\title{
GRAVITONS, DARK MATTER, AND CLASSICAL GRAVITATION
}

\author{
Marcelo Samuel Berman ${ }^{1}$ \\ ${ }^{1}$ Instituto Albert Einstein / Latinamerica - Av. Candido Hartmann, 575 - \# 17 and \\ 80730-440 - Curitiba - PR - Brazil email: msberman@institutoalberteinstein.org
}

(Dated: (Last Version: 16 July, 2009))

\begin{abstract}
We find that the quantum of gravity, the graviton, has time-varying mass (the gomidium), and radius (the somium); both vary with $R^{-1}$; and its frequency is given by Hubble's parameter. Dark matter can be made of such gravitons. The number of gravitons varies with $R^{2}$. The Machian radiated power of the Universe leads us to a "correct" energy density for the gravitons as dark matter.
\end{abstract}




\section{GRAVITONS, DARK MATTER, AND CLASSICAL GRAVITATION}

\section{MARCELO SAMUEL BERMAN}

Berman (2007, 2007a, 2007b, 2008), has suggested that the relative contributions to the total energy of the Universe, as composed by inertial mass-energy, gravitational constant, and spin of the Machian Universe, rest unchanged through its history.

According to Heisenberg's uncertainty principle, any two conjugate quantities, in the sense of Hamilton's canonical ones, carry uncertainties, $\Delta Q$ and $\Delta P$, which obey the condition (Leighton, 1959),

$$
\Delta Q \Delta P \gtrsim h
$$

If we consider maxima $\Delta P$, we obtain minima $\Delta Q$.

If $\Delta P$ stands for the uncertainty in linear momentum, given, say, by the product of mass and speed, then, its maximum value must be the product of the largest mass in the Universe by the largest speed,

$$
\Delta P=M_{U} c
$$

We thus, obtain a minimum length value,

$$
\Delta Q \approx \frac{h}{c M_{U}}
$$

When we plug the mass of the Universe $10^{56}$ grams, we find, what we call the minimal length in the present Universe ( somium , after M.M. Som):

$$
\Delta Q \approx \Delta l=10^{-92} \mathrm{~cm}
$$

Now, let us think of the largest time (age) in the Universe,

$$
t_{U} \approx 10^{10} \text { years. }
$$


Its conjugate variable, will point out to a minimum inertial energy and a minimum inertial mass $(\Delta m)$,

$$
\Delta E=c^{2} \Delta m \approx \frac{h}{t_{U}}
$$

We find, then, the minimal mass in the Universe, which we associate with the mass of present day quantum, which we term the gomidium (after F.M. Gomide):

$$
\Delta m \approx 10^{-65} \text { grams }
$$

Gomide (1963) has found the above mass, in other schema.

Analogously, we could repeat the calculation for Planck's mass, and we then would obtain numerically the same values attained by gomidia and somia in Planck's Universe, which coincide with Planck's length and Planck's time:

$$
\begin{aligned}
& M_{P l} c \Delta l_{P l}=\frac{h}{2 \pi} \\
& M_{P l} c^{2} \Delta t_{P l}=\frac{h}{2 \pi}
\end{aligned}
$$

The two above relations, arise from their respective Planck definitions.

These are the values of Planck's time gomidium $\left(M_{P l}\right)$, and somium $\left(\Delta l_{P l}\right)$. We must yet put a law for the time variation of gomidia and somia. We recognize that the relation between the gomidium values for the present Universe, and for Planck's one, are related by:

$$
\frac{\Delta m}{M_{P l}} \approx\left(\frac{R}{\Delta l_{P l}}\right)^{-1} \approx 10^{-60}
$$

On the other hand, we find the ratio between the somium for the present Universe, with the Planck's length,

$$
\frac{\Delta l}{\Delta l_{P l}} \approx \frac{\Delta l_{P l}}{R} \approx 10^{-60}
$$

We have found that the gomidium and the somium, depend on $R^{-1}$. Consider now that the Universe has a spin, L (Berman, 2007b, 2008, 2008a, 2008b). For Planck's time, we have only one possibility, 


$$
L_{P l}=\frac{h}{2 \pi} \quad .
$$

An expression for the spin of the Universe, at any time, that would reduce to the above Planck's value for Planck's time, is given by:

$$
L=M R c
$$

Berman (2007, 2007a, 2008) has shown that as a result of the Machian hypothesis above, the radius of the Universe would be linearly proportional to its mass. This being the case, the last relation implies that,

$$
L \propto R^{2}
$$

Consider now that all the gravitons of cosmological origin, are aligned with the spin of the Universe, due to a universal magnetic field. If the number of such particles performs a total spin given by $L$, we would find the number of such particles,

$$
n \propto R^{2}
$$

From the above, we find the energy density of the hypothetical gravitons,

$$
\rho=\frac{n \Delta m}{\frac{4}{3} \pi R^{3}} \propto R^{-2}
$$

We hint that the gravitons with such mass, will perform the task of dark matter in the Universe. The numerical values of $\rho$, can be compared with the inertial density in the Universe,

$$
\rho_{i}=\frac{M}{\frac{4}{3} \pi R^{3}} \propto R^{-2}
$$

If $\rho_{i}$ amounts to $5 \%$ of the critical energy, and $\rho$ equals $27 \%$, the same $R^{-2}$ dependence, makes the relative contributions from dark matter (i.e. the gravitons) and visible mass (inertial), become constant over history.

If the graviton has a frequency $\nu$, its energy is given by,

$$
E=\frac{h}{2 \pi} \nu=\Delta m c^{2}
$$


For the present Universe, we would find:

$\nu \approx 10^{-18} \mathrm{sec}^{-1}$

This makes for Hubble's constant.

Consider now the Machian picture of the cosmological gravitons energy density. From the theory of gravitational radiation, we know that, for a spinning "sphere", oblated, the power of radiation is given by Einstein's quadrupole formula,

$$
P=\frac{d E}{d t}=-\frac{G}{5 c^{5}} \sum \dddot{Q}_{\alpha \beta}^{2}
$$

where $\dddot{Q}_{\alpha \beta}^{2}$ stands for the quadrupole moment of the mass distribution. For a periodic rotation, with Machian angular speed,

$$
\omega=\frac{c}{R}
$$

relation (20) will be given by,

$$
P \approx \frac{c^{5}}{G}\left(\frac{G M}{c^{2} R}\right)^{2} \approx \frac{c^{5}}{G}=10^{52} \text { watts }
$$

because of the Brans-Dicke relation $\frac{G M}{c^{2} R} \approx 1$.

Again, for a Machian Universe, we may take the radius definition,

$$
R \approx c T
$$

where $T$ is Hubble's time; we find then, the mass density,

$$
\rho=\frac{c^{4}}{G} R^{-2}
$$

If we plug $R \cong 10^{28} \mathrm{~cm}$, we shall find that the mass energy density of the cosmological gravitons is given by the correct order of magnitude of dark matter density.

Summarizing, Heisenberg's uncertainty principle, yields the quanta of length and mass, respectively, the somium and gomidium, which we associate with the graviton, and dark matter. For Planck's Universe, the minimal length, mass and spin, are given by Planck's 
values. We are in face of time-varying gravitons. The mass of such gravitons, may not be rest one, but the equivalent of mass-energy given by the product of Planck's constant and a frequency characteristic of the graviton, given by Hubble's constant for the present Universe.

\section{Acknowledgments}

The author expresses his recognition to his intellectual mentors, friends and colleagues, M.M. Som (deceased) and F.M. Gomide. He thanks the many other colleagues that collaborate with him. The typing was made by Marcelo F. Guimarães, who I consider a friend and to whom my thanks are due for this and many other collaborations. And, last, but not least, I mention the encouragement by Albert, Paula and Geni.

\section{References}

Berman,M.S. (2007) - Introduction to General Relativity, and the Cosmological Constant Problem, Nova Science, New York..

Berman,M.S. (2007a) - Introduction to General Relativistic and Scalar-Tensor Cosmologies, Nova Science, New York.

Berman,M.S. (2007b) - The Pioneer anomaly and a Machian Universe. Astro-

physics and Space Science, 312, 275. See a previous version in Los Alamos Archives, http://arxiv.org/abs/physics/0606117

Berman,M.S. (2008) - A Primer in Black Holes, Mach's Principle and Gravitational Energy, Nova Science, New York.

Berman,M.S. (2008a) - A General Relativistic Rotating Evolutionary Universe, Astrophysics and Space Science, 314, 319-321.

Berman,M.S. (2008b) - A General Relativistic Rotating Evolutionary Universe - Part II, Astrophysics and Space Science, 315, 367-369. Posted with another title, in Los Alamos Archives http://arxiv.org/abs/0801.1954.

Gomide, F.M.(1963) - Nuovo Cimento, 30, 672.

Leighton, R.B. (1959) - Principles of Modern Physics, McGraw-Hill, N.Y. 